\title{
(5) Rickettsial Infection and Host Reaction, Especially Immune Mechanisms of Tsutsugamushi Disease
}

\author{
Yuzuru Kobayashi, MD \\ The First Department of Internal Medicine, Faculty of Medicine, \\ Ehime University, Ehime
}

Rickettsiae are obligatory intracellular parasites and representative organisms of persistent infection. The rickettsiae remain viable somewhere in host cells for months or years after the recovery from infection. Thus the host-parasite relationship has preserved the balance for a long time. However, the balance between the immune mechanism and the parasite is disturbed somehow, and a recrudescence occurs. For instance, Brill-Zinsser disease has been known as a recrudescence of epidemic typhus after the primary attack.

From this point of view, we studied on the immune mechanisms of host with rickettsial infection.

1. Analysis of lymphoid cell subpopulations of the spleens of mice infected with Rickettsia tsutsugamushi. When mice infected with Gilliam strain of high virulent $R$. tsutsugamushi, the number of lymphoid cells, both $\mathrm{T}$ and $\mathrm{B}$ cells, increased transiently at the time of manifestation of symptoms, and decreased later at the critical stage. But, in the long-survived mice, $\mathrm{T}$ cells increased first prior to the convalescent stage, whereas, increase of B cells occurred when the mice recovered. Serum antibodies were detected at the second week after the infection. They reached to high titers at the fourth week and were maintained for a long time.

These findings suggested that increase of $T$ cells had a very important role on recovering from rickettsial infections.

2. Effect of cyclophosphamide against mice infected with R. tsutsugamushi. Both high and low virulent strains of $R$. tsutsugamushi multiplied extraordinarily well in cyclophosphamide-treated mice. In particular, the untreated mice infected with Irie strain of low virulent $R$. tsutsugamushi progressed subclinically, but the treated mice infected with the Irie strain showed ruffled fur, weakness at the last stage and died, and almost all mononuclear cells in peritoneal fluid were filled with rickettsiae and also a tremendous number of free rickettsiae outside of the cells was frequently seen. Serum antibodies were detected in the cyclophosphamide-treated mice at the third week after infection.

3. Immune response of congenitally athymic nude mice infected with $R$. tsutsugamushi. Athymic mice infected with both high and low virulent strains of $R$. tsutsugamushi were taken ill and died. The pathological findings were similar to those of normal mice infected with the high virulent Gilliam strain. Rickettsial particles were numerous in peritoneal smears of the infected athymic mice. Particularly, the findings of athymic mice infected with the low virulent Irie strain presented striking contrast to those of the infected normal mice.

Tetracycline was effective for the treatment of both athymic and normal mice infected with $R$. tsutsugamushi. When the normal mice infected with Gilliam strain were treated with tetracycline for 5-7 days in the acme of illness, the mice recovered without recrudescence and were highly immunized. On the other hand, although tetracycline therapy was effective for prolonging the life of athymic mice, they showed little progress toward recovery and were recrudesced by discontinuance of the therapy, finally died. Moreover, the serum antibody titer from infected athymic mice was negative even at the 55 th day after infection.

4. Effect of $T$ cells transfer to mice infected with $R$ tsutsugamushi. $\mathrm{T}$ cells were purified from the spleens of normal mice immunized with the high virulent Gilliam strain by nylon wool column, and were transferred to both normal and athymic mice infected with the homologous strain. The infected normal and athymic mice were survived by the treatment with the immune mouse $\mathrm{T}$ cells, whereas the untreated normal control mice in- 
fected with the Gilliam strain were died. And the infected athymic mice treated with $\mathrm{T}$ cells from non-immune normal mice were also taken ill and died. On the other hand, the athymic mice infected with the low virulent Irie strain were survived by the treatment with both immune and non-immune mouse $T$ cells. The serum antibodies were recognized from the survived mice.

It was suggested that the progress of rickettsial infection was protected by construction of cellular immune system.

Moreover, appearance and duration of protective effect of $\mathrm{T}$ cells from mice after immunization were observed. $T$ cells from mice immunized 7 and 10 days after with the Gilliam strain conferred no protection against the mice infected with the homologous strain. At 14 day postimmunization, the infected mice became ill, but all of the normal mice and a part of the athymic mice were survived. Practically complete protection against lethal infection was observed from days 21 for normal mice and 28 for athymic mice to more than month 12 .

5. Effect of anti-Thy-1, anti-Lyt-1 and antiLyt-2 alloantiserum treatment against immune mouse $T$ cells. In the BALB/c mouse strain, codes for lymphocytes cell membrane alloantigenic determinants are Thy-1.2, Lyt-1.2 and Lyt-2.2. Then, the immune mouse $\mathrm{T}$ cells were treated with anti-Thy-1.2, anti-Lyt-1.2 or anti-Lyt-2.2 alloantiserum for $60 \mathrm{~min}$. at $4^{\circ} \mathrm{C}$ respectively, following that the $\mathrm{T}$ cells were treated with rabbit complement for $60 \mathrm{~min}$. at $37^{\circ} \mathrm{C}$. These immune
$\mathrm{T}$ cells treated with the alloantiserum ana complement were transferred to athymic mice infected with Gilliam strain. The infected athymic mice transferred immune $\mathrm{T}$ cells treated with antiThy-1 or anti-Lyt-1 alloantiserum showed pathological symptoms and died at the same time as the control mice which did not receive immune mouse $\mathrm{T}$ cells. On the other hand, the transfer of immune moue $T$ cells treated with anti-Lyt- 2 alloantiserum gave the mice a longer existance for a few days, but the mice were taken ill and finally died.

These findings showed that inactivation of $T$ cells meant destruction of immunological defensive mechanism in the rickettsial infection. Particularly, helper $\mathrm{T}$ cells sensitive to anti-Lyt-1 alloantiserum seem to play the most important role, but the function was restricted without suppressor or cytotoxic $\mathrm{T}$ cells sensitive to antiLyt-2 alloantiserum.

6. Effect of transfer of immune mouse serum to infected mice. Immune mouse serum against Gilliam strain was transferred intraperitoneally twice to athymic and normal mice at the same time as infection $(0.5 \mathrm{ml})$ and 7 days after infection $(0.2 \mathrm{ml})$. The normal mice became ill, but survived. On the other hand, the athymic mice were died.

\section{CONCLUSION}

It may be concluded that inhibition of progress of tsutsugamushi disease was principally due to cellular immune mechanisms and production of serum antibody was thymus dependency.

\title{
(Supplementary) Macrophage Subsets and Intracellular Fate of Listeria Monocytogenes
}

\author{
Tetsuhei OGawa, MD \\ Department of Internal Medicine, School of Medicine, \\ Keio University, Tokyo
}

Listeria monocytogenes is a facultative intracellular bacterial parasite which replicates within the macrophages of infected host. Acquired cellular resistance to $\mathrm{L}$. monocytogenes requires sensitization of specific T-lymphocytes and subsequent macrophage activation (Mackaness 1969). The purpose of this investigation was to characterize the macrophage subsets in the peritoneal 